\title{
Efecto del ejercicio preprandial sobre el metabolismo intermediario basal y postprandial en pacientes con diabetes tipo 2 de nivel del mar Effect of preprandial exercise on the basal and postprandial intermediary metabolism in patients with type 2 diabetes at sea level
}

\author{
Fausto Garmendia ${ }^{1, a}$, Rosa Pando ${ }^{1,2, b}$, William Torres ${ }^{3, c}$, Wuili Valqui, \\ ${ }^{1}$ Instituto de Investigaciones Clínicas, Facultad de Medicina, Universidad Nacional Mayor de San Marcos. Lima, Perú. \\ ${ }^{2}$ Servicio de Endocrinologia, Hospital Nacional Dos de Mayo. Lima, Perú. \\ ${ }^{3}$ Departamento de Medicina, Hospital Nacional Dos de Mayo. Lima, Perú. \\ ${ }^{a}$ Profesor Extraordinario Experto, ORCID: https://orcid.org/0000-0002-6513-8743 \\ ${ }^{\mathrm{b}}$ Doctora en medicina, ORCID: https://orcid. org/0000-0002-6119-802X \\ ${ }^{\circ}$ Médico cirujano, ORCID: https://orcid.org/0000-0003-3379-2211 \\ ${ }^{d}$ Médico cirujano, ORCID: https://orcid.org/0000-0001-7817-4252
}

An Fac med. 2019;80(2):173-6 / DOI: https://10.15381/anales.802.16046

\section{Correspondencia:}

Fausto Garmendia Lorena

garmendiafausto@gmail.com

Recibido: 3 de abril 2019

Aceptado: 24 de mayo 2019

Publicación en línea: 28 de junio 2019

Conflictos de interés: Los autores declaran no tener conflictos de interés

Fuente de financiamiento: FEDU, Universidad Nacional Mayor de San Marcos

Citar como: Garmendia F, Pando R, Torres W, Valqui W. Efecto del ejercicio preprandial sobre el metabolismo intermediario basal y postprandial en pacientes con diabetes tipo 2 de nivel del mar. An Fac med. 2019; 80(2):173-6. DOI: https:// 10.15381/anales.802.16046

\section{Resumen}

Introducción. Los pilares terapéuticos del control metabólico de la diabetes mellitus tipo 2 (DM2) son el régimen alimenticio y la actividad física. Objetivo. Estudiar el efecto del ejercicio preprandial de corta duración y ocasional sobre el metabolismo intermediario basal y postprandial en personas con DM2. Métodos. Estudio experimental, transversal, en 16 mujeres y 15 varones con DM2, de nivel del mar, de 40 a 68 años, sometidos a dos pruebas, basal (A) y a post-ejercicio preprandial (B), consistente en una caminata durante 30 minutos a $4 \mathrm{Km} / \mathrm{hora}$ en faja sin fin. En ayunas y luego de un desayuno con $730 \mathrm{Kcal}$., $55,4 \%$ de grasas, $37,2 \%$ de hidratos de carbono y $7,34 \%$ de proteinas, se midió en sangre, glucosa, colesterol total, colesterol HDL, triglicéridos, insulina y ácidos grasos no esterificados (AGNE) durante 6 horas. El colesterol VLDL, LDL NoHDL y HOMA fueron calculados. Resultados. El ejercicio determinó una disminución de las concentraciones de glucosa, insulina y AGNE en la fase postprandial. Conclusión. El ejercicio mejoró el control metabólico postprandial en DM2.

Palabras clave: Diabetes Mellitus; Ejercicio; Metabolismo Basall.

\section{Abstract}

Introduction. The therapeutic support of the metabolic control of diabetes mellitus type 2 (DM2) are the diet and physical activity.Objetive: To study the effect of a short and casual exercise on the basal and postprandial intermediary metabolism of individuals with DM2. Methods. A total of 16 female and 15 male, DM2 patients, 40 to 68 years old, were included in a experimental, tranversal study. They were submitted to a basal (A) and post-exercise (B) tests, the last one consisting in 30 minutes walking at $4 \mathrm{Km} /$ hour on a treadmill, Under fasting, state, glucose, total cholesterol, HDL cholesterol, triglycerides, insulin and non-sterified fatty acids (NEFA) were measured in blood. Cholesterol VLDL, LDL, Non-HDL and HOMA were calculated. After a breakfast consisting in 55,5\% fat, 37,2\% carbohydrates, 7,34\% proteins containing $730 \mathrm{Kcal}$, glucose, insulin, triglycerides and NEFA were measured during 6 hours. Results: The exercise produced a lowering of glucose, insulin and NEFA during postprandial state. Conclusion: The exercise ameliorates the metabolic control at postprandial state of DM2.

Keywords: Diabetes mellitus type 2, exercise, basal metabolis, postprandial, metabolism. 


\section{INTRODUCCION}

Los pilares terapéuticos del control metabólico de la diabetes mellitus tipo 2 (DM2) son el régimen alimenticio y la actividad física ${ }^{1}$. El régimen alimenticio está orientado a obtener un peso adecuado y a evitar variaciones bruscas de la glicemia. Se ha demostrado que la actividad física ocasiona, por una vía independiente de la insulina, una mayor eficiencia de los receptores de insulina y que activa la movilización de las proteínas trasportadoras de glucosa (Glut 4) ${ }^{2}$.

Los tipos de actividad física que se ha empleado para el control metabólico de la DM2 han sido de muy diversa naturaleza: aeróbica, anaeróbica, casual o aguda, sostenida, de estiramiento, mixta; si bien los estudios muestran una mejoría significativa en el control metabólico, los resultados fueron de grado variable en protocolos estructurados temporales que no se acercan a la realidad de la vida cotidiana de los pacientes ${ }^{3-6}$. Como la diabetes es una enfermedad crónica, la actividad física debe ser constante e igual a la de personas no diabéticas, de acuerdo a su estado de salud, capacidad física, actividad física previa, condiciones laborales y otras variables, por lo que se hace necesario prescribirla en forma individual.

Para la presente investigación planteamos que el ejercicio de ligera intensidad, casual y con una sobrecarga alimenticia similar a la de la vida cotidiana, tendría un efecto beneficioso sobre el control metabólico de la DM2. Así, el objetivo fue estudiar el efecto del ejercicio preprandial, de corta duración, casual y con una sobrecarga alimenticia similar al de la vida cotidiana, sobre el control metabólico de personas con DM2.

\section{MÉTODOS}

Se realizó un estudio experimental, transversal, en un grupo de 31 personas con DM2, residentes a nivel del mar: 16 mujeres y 15 varones, de 40 a 68 años de edad, con un tiempo de enfermedad no mayor de cinco años, sin manifestaciones crónicas de la DM2. En una ficha clínica se registró información respecto a su filiación, historia actual, antecedentes personales fisiológicos, patológicos y antecedentes familiares.
La actividad física previa al estudio fue catalogada en sedentaria o baja (realizada para las actividades primordiales), media (5 o más días de actividad física de intensidad moderada o caminar por lo menos 30 minutos) y alta (7 o más días de cualquier combinación de actividad física leve, moderada o vigorosa que alcance un registro de 3.000 METs-min/ semana1-2,9 km diarios) $)^{7}$. Los pacientes fueron sometidos a dos pruebas: basal (A) y post-ejercicio (B), en ambas se midieron los marcadores bioquímicos en ayunas y durante las 6 horas posteriores a la ingesta de un desayuno similar al de la vida cotidiana que contenía $730 \mathrm{Kcal}$ y compuesta de: 64 g de grasas $(55,4 \%), 68$ g de hidratos de carbono $(37,2 \%)$ y 13,4 g de proteínas (7,34\%) estandarizado de acuerdo a la propuesta de Garmendia ${ }^{8}$. El ejercicio físico fue preprandial.

Se determinaron los perfiles antropométricos de los participantes del estudio. La actividad física consistió en una caminata ligera durante 30 minutos a una velocidad de $4 \mathrm{~km} /$ hora en una faja sin fin equivalente a 3 METs. Entre ambas pruebas hubo una diferencia no mayor de dos

Tabla 1. Características sociodemográficas, antropométricas y metabólicas basales de pacientes con diabetes mellitus tipo 2 y residentes a nivel del mar

\begin{tabular}{|c|c|c|c|}
\hline & Mujeres & Varones & p \\
\hline $\mathrm{n}$ & 16 & 15 & \\
\hline Edad (años) & $54,2 \pm 8,04$ & $54,2 \pm 10,2$ & 0,996 \\
\hline Peso (Kg) & $59,0 \pm 0,22$ & $71,6 \pm 8,96$ & 0,001 \\
\hline Talla (m) & $1,49 \pm 0,06$ & $1,64 \pm 0,07$ & 0,000 \\
\hline IMC (peso/talla²) & $26,4 \pm 3,05$ & $26,5 \pm 2,60$ & 0,906 \\
\hline $\mathrm{CA},(\mathrm{cm})$ & $93,7 \pm 7,57$ & $95,6 \pm 6,15$ & 0,465 \\
\hline PAS (mmHg) & $123,3 \pm 17,0$ & $120,0 \pm 15,0$ & 0,589 \\
\hline PAD (mmHg) & $72,5 \pm 9,30$ & $75,8 \pm 11,0$ & 0,368 \\
\hline $\mathrm{CT}(\mathrm{mg} / \mathrm{dL})$ & $213,6 \pm 53,5$ & $179,8 \pm 32,3$ & 0,042 \\
\hline $\mathrm{HDL}(\mathrm{mg} / \mathrm{dL})$ & $37,6 \pm 8,96$ & $29,6 \pm 8,55$ & 0,017 \\
\hline $\mathrm{Tg}(\mathrm{mg} / \mathrm{dL})$ & $177,6 \pm 111,1$ & $180,1 \pm 112,0$ & 0,952 \\
\hline $\operatorname{VLDL}(\mathrm{mg} / \mathrm{dL})$ & $32,7 \pm 17,2$ & $32,3 \pm 18,9$ & 0,948 \\
\hline $\mathrm{LDL}(\mathrm{mg} / \mathrm{dL})$ & $148,4 \pm 42,6$ & $119,4 \pm 35,6$ & 0,056 \\
\hline NoHDL (mg/dL) & $175,9 \pm 50,3$ & $150,1 \pm 30,5$ & 0,095 \\
\hline Glucosa (mg/ dL) & $157,2 \pm 67,4$ & $116,6 \pm 48,8$ & 0,064 \\
\hline HbA1c (\%) & $11,3 \pm 3,33$ & $9,70 \pm 2,75$ & 0,140 \\
\hline Insulina (uUI/mL) & $7,76 \pm 4,23$ & $9,10 \pm 6,44$ & 0,504 \\
\hline HOMA & $2,85 \pm 1,68$ & $2,67 \pm 2,22$ & 0,748 \\
\hline AGNE (mEq/L) & $487,0 \pm 132,7$ & $446,9 \pm 188,2$ & 0,502 \\
\hline
\end{tabular}

IMC: índice de masa corporal; CA: circunferencia abdominal; PAS: presión arterial sistólica; PAD: presión arterial diastólica; HbA1c: hemoglobina glicosilada; HOMA: índice de resistencia a la insulina 


\section{RESULTADOS}

En la tabla 1 se describen las características generales de la población estudiada en el momento de su ingreso al estudio. Así, la edad fue semejante, los varones tuvieron mayor peso y talla que las mujeres, el índice de masa corporal (IMC) fue semejante $y$, aparte del CT y HDL mayores en las mujeres no se encontró otras diferencias, incluyendo el estado del control metabólico.

La mayoría de los pacientes 18 (58\%) eran sedentarios, $12(38,7 \%)$ realizaban actividad física media y $1(3,22 \%)$ alta, antes de ingresar al estudio. En la comparación de las áreas sobre la basal de las pruebas A y B del período postprandial, se encontraron diferencias significativas para las concentraciones de glucosa, insulina y AGNE como se aprecia en las figuras 1, 2, y 3. Si bien los Tg disminuyeron con el ejercicio, las diferencias no fueron estadísticamente significativas, según se muestra en la tabla 2. No se encontró diferencias significativas en la comparación de los resultados en función de la actividad física previa al estudio realizada por los pacientes.

Tabla 2. Comparación de las áreas sobre la basal, de las concentraciones de triglicéridos en el período postprandial de pacientes con diabetes mellitus tipo 2 sometidos a ejercicio preprandial.

\begin{tabular}{lcccccc} 
Minutos & $\mathbf{3 0}$ & $\mathbf{6 0}$ & $\mathbf{1 2 0}$ & $\mathbf{2 4 0}$ & $\mathbf{3 6 0}$ & Suma \\
Basal & $5,06 \pm 41,3^{*}$ & $19,9 \pm 60,1$ & $51,4 \pm 50,8$ & $84,1 \pm 47,4$ & $86,5 \pm 66,2$ & $247,1 \pm 167,8$ \\
\hline Post-ejercicio & $-6,29 \pm 24,2$ & $24,9 \pm 49,9$ & $40,1 \pm 53,3$ & $65,0 \pm 53,1$ & $69,4 \pm 65,9$ & $235,0 \pm 188,3$ \\
\hline$p$ & 0,154 & 0,720 & 0,376 & 0,087 & 0,135 & 0,779 \\
\hline
\end{tabular}

*Promedio \pm desviación estándar en $\mathrm{mg} / \mathrm{dL}$

\section{DISCUSIÓN}

La disminución significativa de las áreas sobre la basal de las concentraciones de G, I y AGNE entre las pruebas $A$ y $B$ del período postprandial fue el hallazgo más destacado de la presente investigación; lo cual demuestra que una actividad física casual, de corta duración y preprandial también puede tener un efecto beneficioso sobre el control de la DM2, en particular frente a la disyuntiva y falta de consenso de cuál es el momento y el tipo de actividad física que se debe realizar y qué composición de la sobrecarga alimenticia se debe administrar ${ }^{11}$.

La meta fundamental para el control del metabolismo de las personas con diabetes es alcanzar cifras normales de la glicemia,

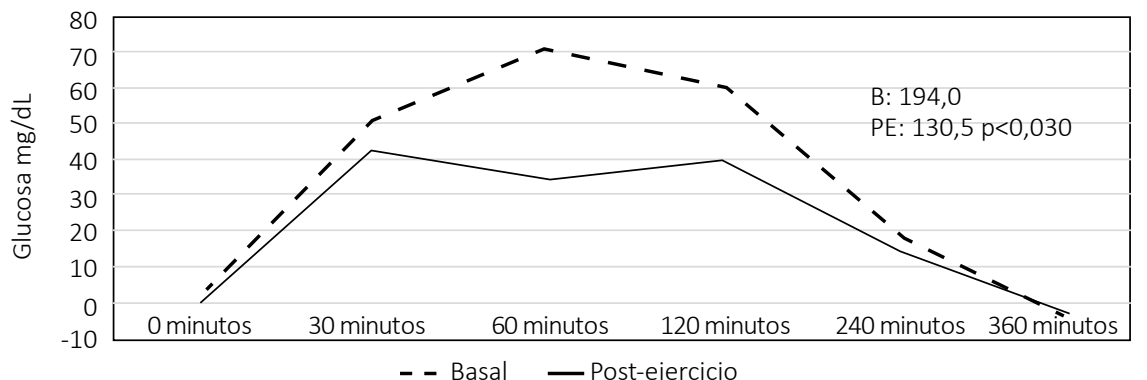

Figura 1. Comparación de las áreas sobre el basal, de las concentraciones de glucosa en el período postprandial de pacientes con diabetes mellitus tipo 2 sometidos a ejercicio preprandial.

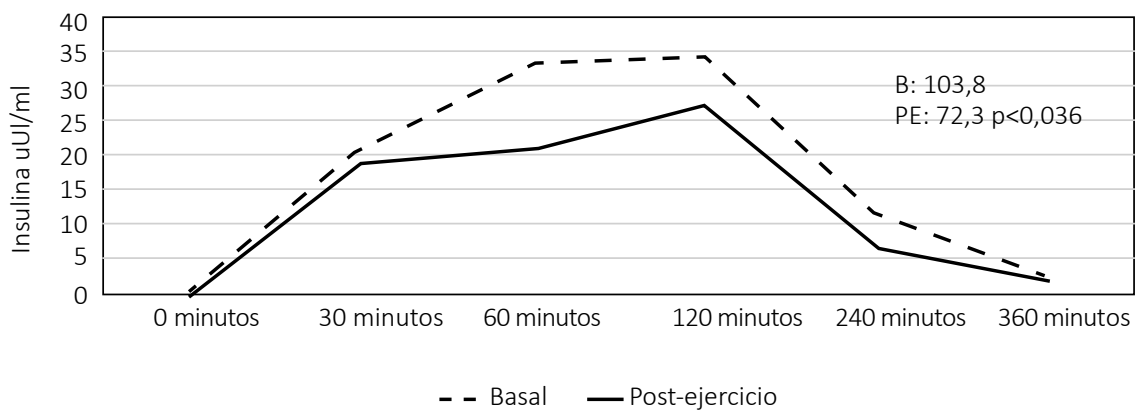

Figura 2. Comparación de las áreas sobre la basal, de las concentraciones de insulina en el período postprandial de pacientes con diabetes mellitus tipo 2 sometidos a ejercicio preprandial. hemoglobina glicosilada menor de 6,5\%, así como el control de la presión arterial y del perfil lipídico. Está ampliamente demostrado que el apropiado control metabólico evita el desarrollo de las manifestaciones crónicas de la DM y con ello una mejor calidad de vida de los pacientes ${ }^{12,13,14}$. Para conseguir ese objetivo, es indispensable que las personas con diabetes adopten un estilo de vida saludable; primero, mediante en un régimen alimenticio que les proporciones un aporte balanceado de macro y micro nutrientes y alcancen un IMC normal y; en segundo lugar, la realización de actividad física regular.

La actividad física debe estar condicionada al estado de salud del paciente, sus preferencias y facilidades. Una de las formas más prácticas, a la que se suelen amoldar la mayoría de los pacientes es la caminata diaria de 3 kilómetros, que puede formar parte de las actividades cotidianas, se la puede efectuar por trechos, no requiere el uso de ropa deportiva, es aeróbica, se puede realizar en cualquier hora del día, puede ser realizada por personas de todas las edades.

Asimismo, se ha demostrado que el cambio a un estilo de vida saludable (régimen alimenticio y actividad física) tiene un efecto preventivo para desarrollar la diabetes en pacientes con prediabetes ${ }^{14}$, disminuye la presión arterial, incrementa la circulación sanguínea, incrementa la secreción de endorfinas y la sensación de bienestar físico y mental, fortalece el sistema músculo-esquelético y favorece la pérdida de peso. Existe suficiente información que demuestra que el ejercicio, de diverso tipo, mejora el control metabólico de pacientes con diabetes mellitus tipo $2^{1,5,6}$. El efecto del ejercicio agudo sobre el control metabólico basal y postprandial de los diabéticos depende del momento en que se efectúa el ejercicio y de la composición de la sobrecarga alimenticia que se propor- 


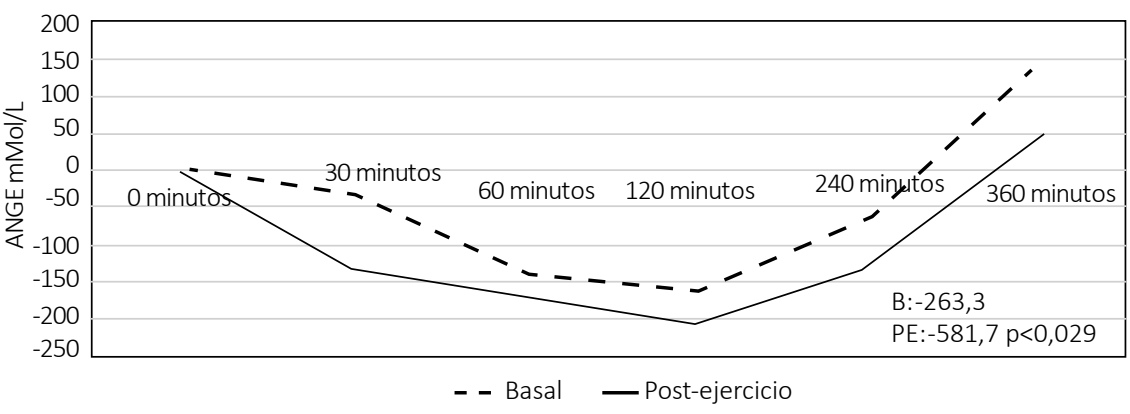

Figura 3. Comparación de las áreas sobre la basal, de las concentraciones de AGNE en el periodo postprandial de pacientes con diabetes mellitus tipo 2 sometidos a ejercicio preprandial.

ciona; así, la realización de ejercicio preprandial y la administración de una sobrecarga alimenticia mixta tienen un mayor efecto sobre la disminución de la glicemia y trigliceridemia ${ }^{15-19}$.

Según nuestros hallazgos, no haber encontrado una disminución significativa de la concentración de triglicéridos por efecto del ejercicio podría explicarse tanto por el hecho que las personas con DM2 que fueron incorporadas en esta investigación, tenían concentraciones basales muy altas y probablemente también por el alto contenido de lípidos de la sobrecarga administrada en el desayuno. Para ambas casos, se ha demostrado que existe un incremento de la respuesta postprandial de triglicéridos ${ }^{20}$.

La disminución del área de la glicemia se explica por el hecho que la actividad física tiene una acción de facilitar el transporte y utilización de la glucosa a nivel celular por una vía independiente de la insulina; es decir, tiene un efecto sinérgico a la insulina. Al disminuir la carga de glucosa al páncreas, se produce una menor secreción de insulina y también una disminución de los AGNE².

Una limitación de nuestro estudio es no haber obtenido información de la variación de la hemoglobina glicosilada (HbA1c); sin embargo, el hecho que las 2 pruebas se efectuaron con un lapso menor de 2 semanas y que el ejercicio no duró más de 30 minutos, hace poco probable que se hubiera encontrado diferencias significativas. Se concluye, según nuestros hallazgos, que la actividad física preprandial, de corta duración y casual, tiene un efecto inmediato de mejorar el control metabólico de personas con DM2 en el período postprandial, con lo que se amplía el conocimiento sobre el efecto del ejercicio sobre el control metabólico de la DM2.

\section{REFERENCIAS BIBLIOGRÁFICAS}

1. Andreus RC, Cooper AR, Montgomery AA, Norcross AJ, Peters TJ, Sharp DJ, et al. Diet or diet plus physical activity versus usual care in patients with newly diagnosed type 2 diabetes: The early ACTID randomised controlled trial. Lancet. 2011; 378:129-39. DOI: https://doi.org/10.1016/S01406736(11)60442-X

2. Hawley JA, Lessard SJ. Exercise traininginduced improvements in insulin action. Acta Physiol. 2008; 192(1): 127-35. DOI: 10.1111/j.17481716.2007.01783.x

3. Sigal RJ, Kenny GP, Wasserman DH, CastanedaSceppa C. Physical activity/exercise and type 2 diabetes. Diabetes Care. 2004; 27 (10): 2518-39. DOI: 10.2337/diacare.27.10.2518

4. Sigal RJ, Kenny GP, Boule NG, Wells GA Prud'homme D, Fortier M, et al. Effects of Aerobic Training, Resistance Training, or Both on Glycemic Control in Type 2 Diabetes. Ann Intern Med. 2007; 147(6): 357-369.

5. Bacchi E, Negri C, Trombetta M, Zanolin ME, Lanza M, et al. Differences in the Acute Effects of Aerobic and Resistance Exercise in Subjects with Type 2 Diabetes: Results from the RAED2 Randomized Trial. PLoS One. 2012; 7 (12): e49937. DOI: 10.1371/journal.pone.0049937

6. Jorge ML, de Oliveira VN, Resende NM, Paraiso LF, Calixto A, Diniz AL, et al. Effects of aerobic, resistance, and combined exercise on metabolic control, inflammatory markers, adipocytikines and muscle insulin signiling in patients with type 2 diabetes mellitus. Metabolism. 2011; 60(9): 1244-52. DOI: 10.1016/j.metabol.2011.01.006

7. Mantilla S, Gómez A. El cuestionario Internacional de Actividad Física. Un instrumento adecuado en el seguimiento de la actividad física poblacional.
Rev Iberoam Fisioter Kinesol. 2007; 10(1): 48-52.

8. Garmendia F, Pando R, Torres W, Valqui W, Jamienson C, Blufstein N. Metabolismo posprandial en adultos mayores normales de nivel del mar. An Fac med. 2003; 64(2): 107-111. DOI: https://doi. org/10.15381/anales.v64i2.1441

9. Friedewald WT, Levy RI, Fredrickson DS. Estimation of the Concentration of Low-Density Lipoprotein Cholesterol in Plasma, Without Use of the Preparative Ultracentrifuge. Clin Chem. 1972; 18(6): 499-502.

10. Matthewus D, Hosker J, Rudenski AS, Naylor BA, Treacher DF, Turner RC. Homeostasis model assessment: insulin resistance and B-cell function from fasting plasma glucose and insulin concentration in man. Diabetologia. 1985; 28 (7):412-419.

11. Haxhi J, Scotto di Palumbo A, Sacchetti M Exercising for metabolic control: is timing important?. Ann Nutr Metab. 2013; 62(1): 14-25. DOI: 10.1159/000343788

12. Diabetes Control and Complications Trial Research Group, Nathan DM, Genuth S, Lachin J, Cleary $\mathrm{P}$, Crofford $\mathrm{O}$, et al. The intensive treatment of diabetes on the development and progression of long-term complications in insulin dependent diabets melltus. N Engl J Med. 1993; 329(14): 977-89. DOI: 10.1056/NEJM199309303291401

13. Ohkubo $Y$, Kishikawa $H$, Araki E, Miyata $T$, Isam $S$, Motoyoshi $S$ et al. Intesive insulin therapy prevents the progression of diabetic microvascular complications in Japanese patients with noninsulin-dependent diabetes mellitus: a randomized prospective 6-years study. Diabetes Research Clin Pract. 1995; 28(2): 103-117.

14. UK Prospective Study (UKPDS) Group. Effect of intensive blood-glucose control with metformine on complications in overweigtht patients with type 2 diabetes (UKPDS 34). Lancet. 1998; 352(9131): 854-65.

15. Knowler WC, Barrett-Connor E, Fowler SE, Hamman RF, Lachin JM, Walker EA, et al. Reduction in the incidence of type 2 diabetes with lifestyle interventrion or metformin. N Engl J Med. 2002; 346 (6):393-403. DOI: 10.1056/NEJMoa012512

16. Boulé NG, Haddad E, Kenny GP, Wells GA, Sigal RJ. Effect of exercise on glycemic control and body mass in type 2 diabetes. A meta-analysis of controIled clinical trials. JAMA. 2001; 286 (10):1218-27.

17. Borror A, Zieff G, Battaglini C, Stoner L. Effects of postprandial exercise on glucose control in individuals with type 2 diabetes: a systematic review. Sports Med. 2018; 48 (6):1479-91. DOI: 10.1007/ s40279-018-0864-X

18. Haxhi J, Leto G, Scotto di Palumbo A, Sbriccoli P, Guidetti L, Fantini $C$ et al. . Exercise at lunchtime: effect on glycemic control and oxidative stress in middle-aged men with type 2 diabetes. Eur J Appl Physiol. 2016; 116 (3):573-582. DOI: $10.1007 /$ s00421-015-3317-3

19. Asano RY, Sales MM, Browne RA, Moraes JF, Coelho Júnior HJ, Moraes MR, et al. Acute effects of physical exercise in type 2 diabetes: A review. World J Diabetes. 2014; 5(5): 659-665. DOI: 10.4239/wjd.v5.i5.659

20. Dubois C, Beaumier G, Juhel C, Armand M, Portugal $\mathrm{H}$, et al. Effect of graded amounts (0-50) of dietary fat on postprandial lipemia in normolipemic adults. Am J Clin Nutr. 1998; 67(1):31-8. DOI: 10.1093/ajcn/67.1.31 